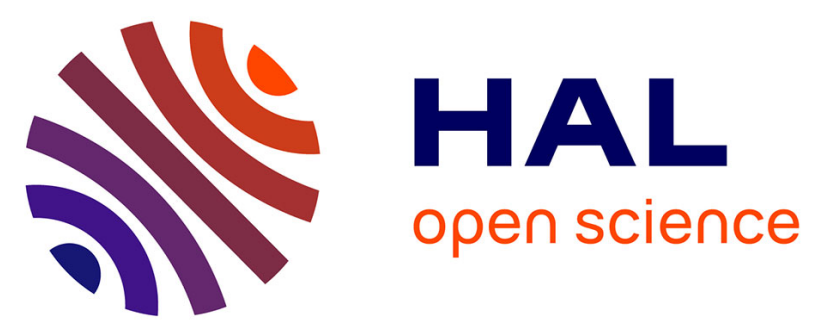

\title{
Hybrid iron oxide-copolymer micelles and vesicles as contrast agents for MRI: impact of the nanostructure on the relaxometric properties
}

\author{
Paolo Arosio, Julie Thevenot, Tomas Orlando, Francesco Orsini, Maurizio \\ Corti, Manuel Mariani, Lorenzo Bordonali, Claudia Innocenti, Claudio \\ Sangregorio, Hugo de Oliveira, et al.
}

\section{To cite this version:}

Paolo Arosio, Julie Thevenot, Tomas Orlando, Francesco Orsini, Maurizio Corti, et al.. Hybrid iron oxide-copolymer micelles and vesicles as contrast agents for MRI: impact of the nanostructure on the relaxometric properties. Journal of materials chemistry B, 2013, 1 (39), pp.5317-5328. 10.1039/C3TB00429E . hal-00959556

\section{HAL Id: hal-00959556 https://hal.science/hal-00959556}

Submitted on 3 Nov 2018

HAL is a multi-disciplinary open access archive for the deposit and dissemination of scientific research documents, whether they are published or not. The documents may come from teaching and research institutions in France or abroad, or from public or private research centers.
L'archive ouverte pluridisciplinaire HAL, est destinée au dépôt et à la diffusion de documents scientifiques de niveau recherche, publiés ou non, émanant des établissements d'enseignement et de recherche français ou étrangers, des laboratoires publics ou privés. 


\title{
Hybrid Iron Oxide-Copolymer Micelles and Vesicles as Contrast Agents for MRI: Impact of the Nanostructure on the Relaxometric Properties
}

\author{
Paulo Arosio $^{\ddagger a}$, Julie Thévenot ${ }^{\sharp b, c}$, Tomas Orlando ${ }^{\ddagger d}$, Francesco Orsini ${ }^{a}$, Maurizio Corti ${ }^{d}$, Manuel \\ Mariani $^{e}$, Lorenzo Bordonali ${ }^{d}$, Claudia Innocenti ${ }^{f}$, Claudio Sangregorio ${ }^{f, g}$, Hugo Oliveira ${ }^{b, c}$, Sébastien \\ ${ }_{5}$ Lecommandoux $^{* b, c}$, Alessandro Lascialfari*a,d,h and Olivier Sandre ${ }^{* b, c}$
}

Received $18^{\text {th }}$ November 2012, Accepted $11^{\text {th }}$ January 2013

DOI: 10.1039/ C3TB00429E

Magnetic resonance imaging (MRI) is at the forefront of non-invasive medical imaging techniques. It provides good spatial and temporal resolution that can be further improved by the use of contrast agents 10 (CAs), providing a valuable tool for diagnostic purposes. Ultrasmall SuperParamagnetic Iron oxides (USPIOs) nanoparticles are attractive MRI contrast agents due to their negative $\left(T_{2}\right)$ contrast enhancement capability and biocompatibility. Clusters of USPIOs with polymer material are of particular interest since they can sustain additional functionalities like drug delivery and targeting. Aiming to establish a relation between the cluster morphology and their efficacy as MRI contrast agent (relaxometric properties), we 15 prepared - by using three different maghemite $\left(\gamma-\mathrm{Fe}_{2} \mathrm{O}_{3}\right)$ USPIOs' diameters - a series of hybrid copolymer/iron oxide CAs presenting two different geometries (micellar or vesicular). The NMR relaxometry profiles confirmed the nature of the physical mechanisms inducing the increased nuclear relaxation rates at low (magnetic anisotropy) and high (Curie relaxation) magnetic fields. A heuristic model, first proposed by Roch, Muller, Gillis, and Brooks, allowed the fitting of the whole longitudinal 20 relaxivity $r_{1}(v)$ profile, for samples with different magnetic core sizes. We show that both types of cluster exhibit transverse relaxivity $\left(r_{2}\right)$ values comparable or higher than those of common contrast agents, over the whole tested frequency range. Moreover, in-depth analysis revealed substantially a linear relation between $r_{2}$ and the number of encapsulated USPIOs divided by the diameter of the clusters $\left(N_{\mathrm{USPIO}} / D_{\mathrm{H}}\right)$, for each USPIOs size. The cluster structure (i.e. micelle or vesicle) appeared to have a mild influence on ${ }_{25}$ the transverse relaxivity value. Indeed, the $r_{2}$ value was mainly governed by the individual size of the USPIOs, correlated to both the cluster external diameter and the magnetic material volume fraction.

\section{Introduction}

The recent years have seen the development of the so-called "theranostic" concept, which purpose is the integration in the 30 same medical device of diagnostic tools and therapeutic agents. More particularly, there was a recent focus on the development of multifunctional nanoparticle drug delivery systems (DDS) that simultaneously serve as medical imaging contrast agents. ${ }^{1}$ Indeed, medical imaging techniques play a key role in clinical 35 diagnostics as they provide spatially resolved anatomical information on diseases. All the current imaging methods (CT, PET/SPECT, MRI, ultrasound imaging, optical imaging) have their advantages and drawbacks/limits, but, among these technologies, MRI stands as one of the best non-invasive imaging 40 modalities thanks to its good spatial and temporal resolution. ${ }^{2-4}$

Magnetic nanoparticles (NPs) have drawn a lot of attention in the biomedicine field ${ }^{5}$ due to their unique magnetic properties and the possibility to functionalize their surface. ${ }^{6,7}$ Thank to their small crystal size, they are magnetic mono-domains with either
45 superparamagnetic or ferromagnetic behavior. Iron oxide crystals (magnetite or maghemite) have been the most extensively studied for biomedical applications. ${ }^{8}$ Magnetic iron oxide phases (ferrites) show a superparamagnetic behaviour for crystal sizes smaller than e.g. $12 \mathrm{~nm}$ (called ultrasmall superparamagnetic iron 50 oxide, USPIO): below this limit, the magnetic moments are free to rotate within the crystal core (Néel's relaxation), so that they do not retain any remanent magnetization in the absence of external magnetic field. When a magnetic field is applied, the propensity of superparamagnetic particles to align their magnetic 55 moment in the direction of the field (i.e. their large magnetic susceptibility) is at the origin of their use as MRI contrast agents. ${ }^{9,10}$ Their magnetic dipoles (arising from electronic spins) interact strongly with the nuclear moments of water protons, enhancing the relaxation rates in tissues where the NPs have 60 accumulated. They predominantly reduce the transverse relaxation time, $T_{2}$ (or $T_{2} *$ in presence of field heterogeneity), of protons, locally lowering the MRI signal intensity as compared to the background signal. Therefore superparamagnetic NPs are most often used as "negative" contrast agents (CAs), in contrast 
with paramagnetic compounds, used as "positive" CAs, because they increase the signal of the tissues. At the bottom of the size scale, ultra-ultrasmall superparamagnetic iron oxide (UUSPIO) cores with diameters below $5 \mathrm{~nm}$ were recently evidenced as ${ }_{5}$ positive CAs (for $T_{1}$-weighted MRI sequences) due to their minimal value of $r_{2} / r_{1}$ as long as clustering is prevented by a repulsive shell. ${ }^{7,11,12}$

Usually, to establish a comparison between MRI CAs, their efficiency as contrast enhancers is measured by the longitudinal 10 (respectively transverse) relaxivity $r_{1}$ (respectively $r_{2}$ ), defined as the relaxation rate $R_{1}=1 / T_{1}$ (respectively $R_{2}=1 / T_{2}$ ) of water protons in presence of CA (minus the value of solvent alone) normalized by the concentration of $\mathrm{CA}$ (in equivalent iron $\mathrm{mmol} \cdot \mathrm{L}^{-1}$ ). Most of the negative MRI CAs developed so far are 15 hybrid nanoparticles (HNPs): ${ }^{13,14}$ these superparamagnetic HNPs consist of an inorganic core made of several iron oxide crystals surrounded by an organic shell - frequently Dextran - leading to a hydrodynamic diameter larger than $40 \mathrm{~nm} .{ }^{1}$ Apart from carbohydrate homopolymers adsorbed by weak H-bonds at the 20 iron oxide surface, other polymers were introduced to prepare HNPs by self-assembly with amphiphilic block copolymers into micelles ${ }^{15}$ or by electrostatic complexation with charged-neutral double-hydrophilic copolymers into polyion complex micelles. ${ }^{16}$ HNPs are of interest for the following reasons: first, the organic 25 part can be loaded with a drug and provide a substrate for (bio)functionalization through a wide range of coupling reactions ${ }^{17}$; then the clustering effect (when several iron oxide crystals are embedded in the organic matrix) both enhances the value of transverse relaxivity ${ }^{15,16}$ and reduces renal clearance as 30 compared to uncoated USPIOs. ${ }^{8,}{ }^{18}$ However, the whole HNP size (including coating) needs to remain below $150-200 \mathrm{~nm}$, because $r_{2}$ starts to decrease for diameters larger than this optimum ${ }^{13}$ and due to sequestration of bigger particles by the mononuclear phagocyte system (MPS). ${ }^{18}$ On the contrary, HNPs with 35 hydrodynamic diameters below $40 \mathrm{~nm}$, made of single USPIOs wrapped by a thin hydrophilic repulsive coating, exhibit rapid renal clearance and thus were introduced mainly for brain tumor studies, when the brain-blood-barrier is damaged. ${ }^{18}$

When designing hybrid nanoparticles (HNPs) as efficient MRI ${ }_{40} \mathrm{CAs}$ from non toxic copolymers loaded with maghemite NPs $(\gamma-$ $\mathrm{Fe}_{2} \mathrm{O}_{3}$ ), numerous parameters remain in the hands of chemists: structure of the cluster, size of the magnetic cores and of the whole objects, composition... The recent concept of theranostic prompted the development of particles with dual functions, acting 45 as both drug delivery system and imaging agent. The numerous nanosized drug delivery systems proposed so far exhibit a variety of morphologies and compositions. One can then wonder if a particular geometry should be preferred in order to obtain high relaxivities: is there any advantage of the micelle geometry (filled 50 sphere) compared to the less commonly used vesicle structure (hollow shell)? Moreover, is it a better option to increase the core sizes of the USPIOs within the HNPs or to use smaller USPIOs that enable higher loadings? Or will intermediate values of both core size and iron content be the best solution?

55 To address these fundamentally relevant questions, two types of self-assembled structures - respectively vesicles and micelles were obtained by a solvent-displacement self-assembly process with two different amphiphilic block copolymers, respectively poly(trimethylene carbonate)-block-poly(L-glutamic acid) ${ }_{60}$ (PTMC-b-PGA) and poly(ethylene glycol)-block-poly $(\gamma$-benzylL-glutamate) (PEG- $b$-PBLG), sharing common features e.g. biocompatibility, biodegradability and presence of one artificially synthesized polypeptide block (respectively PGA and PBLG). In previous studies, we already evidenced the formation of magnetic ${ }_{65}$ vesicles with PGA as hydrophilic block, ${ }^{19}$ and magnetic micelles with PBLG as hydrophobic block. ${ }^{20}$ Measurements of the NMRDispersion (NMRD) curves of the nuclear longitudinal $\left(r_{1}\right)$ and transverse $\left(r_{2}\right)$ relaxivities are reported, from very low frequency to high frequencies used by clinical imagers $(21 \mathrm{MHz}$ and ${ }_{70} 64 \mathrm{MHz}$ ). We show here that both types of HNPs behave like efficient "negative" contrast agents increasing the transverse relaxivity of the protons. They offer improved properties as negative CAs (higher $r_{2}$ and $r_{2} / r_{1}$ ) as compared to Endorem ${ }^{\circledR}$, a standard superparamagnetic agent composed of maghemite NPs 75 clustered by Dextran yielding aggregates with undefined shapes and diameters ranging from 50 to $100 \mathrm{~nm} .^{21,22}$ Furthermore, we demonstrate that the (micellar or vesicular) morphology has a lower impact on the transverse relaxivities than the core size of the maghemite crystals to obtain the best relaxometric properties. ${ }_{80}$ Following a universal method to derive the transverse relaxivity from the outer size of the HNP, its magnetization, and the internal iron oxide volume fraction, ${ }^{23}$ we evidence here that the relevant parameter for $r_{2}$ is the number of encapsulated USPIOs normalized by the hydrodynamic diameter, whenever the particle 85 is a micelle or a vesicle.

\section{Experimental section}

\section{Reference material}

Used as reference material, Endorem ${ }^{\circledR}$ was purchased from Guerbet Group (Roissy, France) and used without further 90 purification. Thermogravimetric analysis (TGA, TA Instruments) yielded an inorganic content of $17.3 \mathrm{~g} \cdot \mathrm{L}^{-1}$ iron oxide, which is coherent with the iron concentration of $11.2 \mathrm{~g} \cdot \mathrm{L}^{-1}\left(200 \mathrm{mM}_{\mathrm{Fe}}\right)$ claimed by the supplier. From the organic content also measured by TGA, iron oxide represents $63.8 \mathrm{wt} \%$ of the total solid matter 95 or, equivalently, a volume fraction of $26 \% \mathrm{v} / \mathrm{v}$ in the composite.

\section{Synthesis of iron oxide superparamagnetic nanoparticles}

Superparamagnetic maghemite $\left(\gamma-\mathrm{Fe}_{2} \mathrm{O}_{3}\right)$ nanoparticles were synthesized in water according to Massart's procedure. ${ }^{24}$ Details of the process are described in Supporting Info. Briefly, 100 magnetite $\mathrm{Fe}_{3} \mathrm{O}_{4}$ nanocrystals (also called ferrous ferrite $\mathrm{FeO} \cdot \mathrm{Fe}_{2} \mathrm{O}_{3}$ ) were prepared from an alkaline coprecipitation of a mixture of iron $+\mathrm{II}$ and iron $+\mathrm{III}$ chloride salts in $\mathrm{HCl}$ solution. After acidification, magnetite was oxidized into maghemite by immersion into boiling ferric nitrate solution. The "ionic 105 ferrofluid" obtained, which presents a wide size-dispersity, was treated with a size-sorting procedure based on fractionated phaseseparation. ${ }^{25}$ After repeating this process three times (see Fig. SI1 in Supporting Information), three fractions with well defined sizes were selected for this work. In order to disperse them in a 110 chlorinated solvent, ${ }^{26}$ these fractions were coated with Beycostat NB09 (CECA, Arkema group, France), an anionic surfactant composed of a mixture of mono- and di-esters of phosphoric acid with alkylphenol chains containing nine ethoxy groups. ${ }^{27}$ The grafting was performed by reacting $200 \mathrm{~g}$ of surfactant per mole 
of iron oxide (around $20 \mathrm{~mol} \%$ ) in acidic conditions $\left(\mathrm{HNO}_{3}\right.$ $2 \mathrm{~mol} \cdot \mathrm{L}^{-1}$ ) for $30 \mathrm{~min}$ at $60{ }^{\circ} \mathrm{C}$ under vigorous stirring and subsequent removal of the surfactant in excess by 5 washing cycles with methanol. The obtained precipitates of hydrophobic ${ }_{5}$ USPIOs were readily suspended in dichloromethane by simple vortexing, leading to the hydrodynamic diameters measured by dynamic light scattering listed in Table SI-I.

\section{Copolymers synthesis}

$\mathrm{PEG}_{45}-b-\mathrm{PBLG}_{13}$ was synthesized according to a protocol 10 described elsewhere with minor modifications. ${ }^{28}$ Briefly, $\mathrm{CH}_{3} \mathrm{O}$ PEG-NH 2 (2000 g. $\mathrm{mol}^{-1}$, RAPP Polymere, Germany; $2 \mathrm{~g}, 1 \mathrm{mM}$ ) was dissolved in $2 \mathrm{~mL}$ dioxane, freeze-dried and dissolved in dry DMF $\left(0.1 \mathrm{~g} \cdot \mathrm{mL}^{-1}\right)$. In a glove-box, $\gamma$-benzyl-L-glutamate $\mathrm{N}$ carboxyanhydride (Isochem, France; $4.20 \mathrm{~g}, 16 \mathrm{mM}$ ) was 15 introduced into a flame-dried Schlenk flask and dissolved in anhydrous DMF $\left(0.1 \mathrm{~g} \cdot \mathrm{mL}^{-1}\right)$. This solution was then added under vacuum to the first flask. The mixture was stirred for 48 hours at $40{ }^{\circ} \mathrm{C}$ in an oil bath. The polymerization medium was concentrated by cryo-distillation and the copolymer was 20 recovered by precipitation into cold diethyl ether. The white powdery solid was then washed three times with diethyl ether and finally dried under dynamic vacuum for 24 hours. The degree of polymerization of PBLG measured by proton NMR was $D P=13 \pm 1$, corresponding to a molar mass of $2.8 \pm 0.2 \mathrm{~kg} \cdot \mathrm{mol}^{-1}$. ${ }_{25}$ For some samples, we used a $1: 1$ mixture of PEG- $b$-PBLG batches with the same polypeptide block length but differing by the molar mass of the PEG chains (respectively 2000 and $\left.12000 \mathrm{~g} \cdot \mathrm{mol}^{-1}\right)$.

$\mathrm{PTMC}_{25}-b$-PGA 12 diblock copolymer was synthesized 30 following a previously published method. ${ }^{29}$ Briefly, amino endfunctionalised PTMC (PTMC- $\mathrm{NH}_{2}$ ) was synthesized by ringopening polymerization (ROP) of TMC (kindly provided as a gift by Labso Chimie Fine, Boerhinger Ingelheim, France) in the presence of diethyl zinc and 3-(Fmoc-amino)-1-propanol as 35 initiator, followed by the deprotection of the amine group with piperidin. PTMC- $b$-PBLG was then prepared by ROP of $\gamma$ benzyl-L-glutamate $\mathrm{N}$-carboxyanhydride (Isochem) initiated by the PTMC- $\mathrm{NH}_{2}$ macroinitiator and subsequently deprotected into PTMC- $b$-PGA amphiphilic diblock copolymer by catalytic 40 hydrogenation.

\section{Preparation of hybrid (copolymer / iron oxide) particles}

Hybrid nanoparticles (HNPs) were obtained by the nanoprecipitation method also called solvent displacement or solvent assisted dispersion. ${ }^{30}$

$45 \quad$ For PTMC- $b$-PGA hybrid vesicles, Tris buffer $50 \mathrm{mM} \mathrm{pH} 7.4$ $(9 \mathrm{~mL})$ was added in 10 seconds under magnetic stirring (500 rpm) at room temperature to a solution in DMSO of PTMC$b$-PGA block copolymer $(10 \mathrm{mg}, 1 \mathrm{~mL})$ and the volume of iron oxide NPs in dichloromethane corresponding to the desired feed 50 weight ratio (FWR, defined as the weight of iron oxide divided by the copolymer weight multiplied by 100). The traces of dichloromethane became negligible after rapid evaporation. The buffering at neutral $\mathrm{pH}$ was necessary to deprotonate the acid moieties of the PGA blocks and provide them negative charges.

${ }_{55}$ In the case of hybrid PEG- $b$-PBLG particles, ultrapure water $(120 \mathrm{~mL})$ was added in 25 seconds under magnetic stirring at room temperature $\left(22{ }^{\circ} \mathrm{C}\right)$ to a solution of PEG- $b$-PBLG block copolymer (45 mg; $40 \mathrm{~mL}$ ) and iron oxide NPs in DMSO. Both solvent and aqueous solutions were filtered $(0.22 \mu \mathrm{m})$ prior to the 60 addition. After nanoprecipitation, DMSO was eliminated from the suspensions by dialysis against ultrapure water for PEG- $b$ PBLG HNPs and Tris $50 \mathrm{mM}$ pH 7.4 for PTMC- $b$-PGA HNPs $(3 \times 5 \mathrm{~L})$.

Total solid content of the final particles dispersions was 65 assessed by differential weighting after water evaporation using a TGA Q50 instrument (TA Instruments).

\section{Particles size and morphology}

Hybrid particles sizes were measured by Dynamic Light Scattering (DLS) using a NanoZS90 apparatus from Malvern 70 Instruments working at a scattering angle of $90^{\circ}$. The sample was kept at constant temperature $\left(25^{\circ} \mathrm{C}\right)$ during all the experiment. Data acquisition was carried out using the automatic adjustment of position, attenuation and measurement time. Three measurements were averaged to obtain mean values of the $\mathrm{Z}$ 75 average hydrodynamic diameter $\left(D_{\mathrm{H}}\right)$ and polydispersity index (PDI) obtained from the ratio of the $2^{\text {nd }}$ order cumulant divided by the square of the $1^{\text {st }}$ order one.

For transmission electron microscopy (TEM), samples were prepared by spraying $1 \mathrm{~g} \cdot \mathrm{L}^{-1}$ suspensions of particles onto a 80 copper grid (200 mesh coated with carbon or carbon/Formvar ${ }^{\mathrm{TM}}$ ) using a homemade spraying tool. After complete drying, samples were stained with a $1 \% \mathrm{w} / \mathrm{v}$ uranyl acetate solution in water. Bright field images were recorded at the Bordeaux Imaging Center (Bordeaux University) on a Hitachi H7650 microscope 85 working at $80 \mathrm{kV}$ and equipped with a GATAN ORIUS SC1100 11 Megapixel camera.

\section{Iron content determination}

Total iron titration providing the value of equivalent ferric ions concentration, $C_{\mathrm{Fe}}$, is of uppermost importance for precise 90 relaxometric studies. An absorption spectrum curve between 200 and $800 \mathrm{~nm}$ wavelengths calibrated by atomic emission spectroscopy was used for rapid and non invasive assessment of iron oxide amount in HNP suspensions (Figure SI-4). To avoid uncertainty coming from a turbidity baseline in case of large size ${ }_{95}$ HNPs inducing a strong light scattering effect, a more precise measurement involving the total disruption of the USPIOs and their conversion into dissolved $\mathrm{Fe}^{3+}$ ions was used. Each aliquot was incubated for 1 hour at $60{ }^{\circ} \mathrm{C}$ in $\mathrm{HCl} 5 \mathrm{~mol} \cdot \mathrm{L}^{-1}$ in a capped vial, and the iron hexachloride complex concentration ${ }_{100} C_{\mathrm{Fe}}=\left[\mathrm{FeCl}_{6}{ }^{3-}\right]$ was read using Beer-Lambert's law at the wavelength of the absorption peak of $\mathrm{FeCl}_{6}{ }^{3-}(350 \mathrm{~nm})$. According to calibration with a series of ferric chloride solutions in $\mathrm{HCl} 5 \mathrm{~mol} \cdot \mathrm{L}^{-1}$ (Figure SI-5), the extinction coefficient of the ferric chloride complex is $\varepsilon_{350 \mathrm{~nm}}=2800 \mathrm{~mol}^{-1} \cdot \mathrm{L} \cdot \mathrm{cm}^{-1}$.

\section{Zero-Field-Cooled/Field-Cooled magnetization curves}

ZFC and FC curves were measured with a SQUID magnetometer (Quantum Design Ltd.) upon increasing temperature, with a $5 \mathrm{mT}$ probing field. Before each procedure, samples were cooled with (FC) or without (ZFC) applying the probe field. The blocking 110 temperatures, $T_{\mathrm{B}}$, identified at the maxima of the $\mathrm{ZFC}$ curves, are reported in Table SI-I. As expected, $T_{\mathrm{B}}$ increases with USPIOs' size: the plot of $25 k_{\mathrm{B}} T_{\mathrm{B}} v s$. volume $\pi d_{\mathrm{w}}{ }^{3} / 6$ leads to an anisotropy constant $K_{\mathrm{a}}=2.4 \times 10^{4} \mathrm{~J} \cdot \mathrm{m}^{-3}$, typical for $\gamma-\mathrm{Fe}_{2} \mathrm{O}_{3}$ (Figure SI-7b). 
Table 1 Characteristics of the studied hybrid PTMC- $b$-PGA vesicles, named according to: V(USPIOs' size)-(theoretical $\gamma$-Fe $\mathrm{F}_{2} \mathrm{O}_{3} \mathrm{FWR}_{\text {) }}$.

\begin{tabular}{|c|c|c|c|c|c|c|c|c|}
\hline \multirow{2}{*}{$\begin{array}{c}\text { Vesicles } \\
\begin{array}{c}\text { Sample } \\
\text { code }\end{array}\end{array}$} & \multicolumn{5}{|c|}{ Inorganic part } & \multirow{2}{*}{$\begin{array}{c}\text { Organic part } \\
\begin{array}{c}\text { Copolymer }] \\
\left(\mathrm{mg} \cdot \mathrm{mL}^{-1}\right)\end{array}\end{array}$} & \multicolumn{2}{|c|}{ DLS results } \\
\hline & USPIOs & $\begin{array}{c}C_{\mathrm{Fe}}(\mathrm{mM}) \\
\text { (spectrum } \\
\text { fit) }\end{array}$ & $\begin{array}{c}C_{\mathrm{Fe}}(\mathrm{mM}) \\
\left(\mathrm{OD}_{350 \mathrm{~nm}}{ }^{\mathrm{c}} \text { after }\right. \\
\text { degradation in }^{\mathrm{H}} \\
\mathrm{HCl})^{\mathrm{a}}\end{array}$ & $\begin{array}{c}\text { Effective } \\
\text { FWR } \\
\text { iron oxide } \\
(\mathrm{wt} \%)\end{array}$ & $\begin{array}{c}\text { Approximate } \\
\text { number of } \\
\text { USPIOs per } \\
\text { HNP }^{\mathrm{d}}\end{array}$ & & $D_{\mathrm{H}}(\mathrm{nm})$ & PDI \\
\hline V6-5 & $6-7 \mathrm{~nm}$ & 0.4 & 0.5 & 5.0 & 22 & 0.8 & 125 & 0.07 \\
\hline V6-10 & $6-7 \mathrm{~nm}$ & 0.8 & 0.95 & 9.5 & 29 & 0.8 & 102 & 0.14 \\
\hline V6-50 & $6-7 \mathrm{~nm}$ & 4.0 & 5.8 & 51.6 & 154 & 0.9 & 109 & 0.11 \\
\hline V6-70 & $6-7 \mathrm{~nm}$ & 6.0 & 7.7 & 68.1 & 235 & 0.9 & 116 & 0.09 \\
\hline V8-35 & $8-10 \mathrm{~nm}$ & 5.4 & 5.9 & 33.8 & 16 & 1.4 & 67 & 0.14 \\
\hline V8-50 & $8-10 \mathrm{~nm}$ & 7.4 & 7.6 & 50.5 & 31 & 1.2 & 79 & 0.15 \\
\hline V10-5 & $10-15 \mathrm{~nm}$ & 0.8 & 0.77 & 5.1 & 1 & 1.2 & 87 & 0.11 \\
\hline V10-10 & $10-15 \mathrm{~nm}$ & 1.5 & 1.4 & 10.1 & 2 & 1.1 & 73 & 0.14 \\
\hline V10-20 & $10-15 \mathrm{~nm}$ & \multicolumn{2}{|c|}{$50^{\mathrm{b}}$} & $20^{\mathrm{b}}$ & 16 & 20.0 & 148 & 0.07 \\
\hline
\end{tabular}

${ }^{a}$ The typical errors for iron assay by UV-V are $\pm 5 \%$ with degradation into ions and only $\pm 15 \%$ without.

${ }^{\mathrm{b}}$ calculated value (experimental value not determined), ${ }^{\mathrm{c}}$ supporting info, ${ }^{\mathrm{d}}$ from (Eq. 2a), assuming a membrane thickness $\delta_{\mathrm{mb}}=10 \mathrm{~nm} .{ }^{30}$

\section{NMR relaxometric measurements}

${ }_{5}$ The ${ }^{1} \mathrm{H}$ NMR relaxometry characterization (NMR-dispersion profile) was performed at physiological $\left(37^{\circ} \mathrm{C}\right)$ and room temperature by measuring the longitudinal and the transverse nuclear relaxation times $T_{1}$ and $T_{2}$, in the frequency range $10 \mathrm{kHz}-60 \mathrm{MHz}$. It should be noticed that the measurements at 10 room and physiological temperatures gave the same results within $10 \%$ (corresponding to viscosity change of water). The range of frequency $v$ has been chosen in order to cover the most used clinical fields i.e. $0.2 \mathrm{~T}(\sim 8.5 \mathrm{MHz}), 0.5 \mathrm{~T}(\sim 21 \mathrm{MHz})$ and $1.5 \mathrm{~T}$ $(\sim 64 \mathrm{MHz})$ and to study the mechanisms that lead to the nuclear 15 relaxation, through the analysis of curves of $r_{1}$ and $r_{2} v s . v$ (NMRD profiles). The NMR signal detection and generation was obtained with a Smartracer ${ }^{\circledR}$ Fast-Field-Cycling relaxometer (Stelar, Mede, Italy) for $10 \mathrm{kHz}-10 \mathrm{MHz},{ }^{31}$ and with a Stelar Spinmaster spectrometer for $v>10 \mathrm{MHz}$. In the second case, 20 standard radio frequency excitation sequences were used for $T_{1}$ and $T_{2}$ measurements, respectively inversion-recovery and Carr Purcell Meiboom Gill (CPMG).

To determine the efficiency of MRI contrast agents, we calculated the longitudinal $\left(r_{1}\right)$ and transverse $\left(r_{2}\right)$ nuclear 25 relaxivities. The relaxivity values are defined as the increase of relaxation rates of the solvent induced by $1 \mathrm{mmol} \cdot \mathrm{L}^{-1}$ of iron:

$$
r_{\mathrm{i}}=\frac{\left(1 / T_{\mathrm{i}}\right)_{\text {meas }}-\left(1 / T_{\mathrm{i}}\right)_{\text {dia }}}{C_{\mathrm{Fe}}}
$$

where $\left(1 / T_{i}\right)_{\text {meas }}$ is the relaxation rate measured for a sample of iron concentration $C_{\mathrm{Fe}}\left(\mathrm{mmol} \cdot \mathrm{L}^{-1}\right)($ see Table 1 and Table 2$)$, and
${ }_{30}\left(1 / T_{i}\right)_{\text {dia }}$ represents the diamagnetic contribution to nuclear relaxation rate of the host solution $\left(\approx 4 \mathrm{~s}^{-1}\right.$ for ultrapure water).

\section{Results and Discussion}

\section{Hybrid particles characterization}

${ }_{35}$ Self-assembly of both copolymers with iron oxide nanoparticles led to stable, well defined, colloidal dispersions in water-based media for the feed weigh ratios (FWRs) indicated in Tables 1 and 2. By carefully choosing the nanoprecipitation parameters, ${ }^{29}$ vesicles of comparable sizes (measured by DLS) were prepared 40 with $\mathrm{PTMC}_{25}-b-\mathrm{PGA}_{12}$ and various amounts of the three USPIOs sizes available (Table 1). By the same nanoprecipitation process, $\mathrm{PEG}_{45}-b-\mathrm{PBLG}_{13}$ formed micelle-like aggregates with the USPIOs (Table 2). These two geometries are depicted schematically on Figure 1. Clearly, for PTMC- $b$-PGA, the 45 amount of USPIO has no impact on the size of the vesicles (as showed in Figure 2 for vesicles loaded with 6-7 nm USPIOs), since the preparation process mainly governs this characteristic. ${ }^{30}$ Indeed, for iron oxide contents ranging from 5 to $70 \%$, the mean size and polydispersity indexes of the vesicles are very similar, 50 with narrow and symmetrical size distributions typical of fairly monodisperse samples. The hydrodynamic diameters and PDI values in a cell culture medium supplemented with up to $10 \%$ foetal bovine serum do not change significantly compared to pure water (in vitro stability and toxicity assays on M10-20 and V105520 will be published in a forthcoming paper). Thus we can infer a good stability of these samples also in vivo. 
Table 2 Characteristics of the studied hybrid PEG- $b$-PBLG micelles, named according to: M(USPIOs' size)-(theoretical $\gamma$ - $\mathrm{Fe}_{2} \mathrm{O}_{3}$ FWR).

\begin{tabular}{|c|c|c|c|c|c|c|c|c|}
\hline \multirow{2}{*}{$\begin{array}{c}\text { Micelles } \\
\begin{array}{c}\text { Sample } \\
\text { code }\end{array}\end{array}$} & \multicolumn{5}{|c|}{ Inorganic part } & \multirow{2}{*}{$\begin{array}{l}\text { Organic part } \\
\qquad \begin{array}{c}{[\text { Copolymer }]} \\
\left(\mathrm{mg} \cdot \mathrm{mL}^{-1}\right)\end{array}\end{array}$} & \multicolumn{2}{|c|}{ DLS results } \\
\hline & USPIOs & $\begin{array}{c}C_{\mathrm{Fe}}(\mathrm{mM}) \\
\text { (spectrum } \\
\quad \text { fit) }\end{array}$ & $\begin{array}{c}C_{\mathrm{Fe}}(\mathrm{mM}) \\
\left(\mathrm{OD}_{350 \mathrm{~nm}}{ }^{\mathrm{c}} \text { after }\right. \\
\text { degradation in } \\
\mathrm{HCl})\end{array}$ & $\begin{array}{c}\text { Effective } \\
\text { FWR } \\
\text { iron oxide } \\
(\mathrm{wt} \%) \\
\end{array}$ & $\begin{array}{c}\text { Approximate } \\
\text { number of } \\
\text { USPIOs per } \\
\text { HNP }^{\mathrm{d}}\end{array}$ & & $D_{\mathrm{H}}(\mathrm{nm})$ & PDI \\
\hline M6-5 & $6-7 \mathrm{~nm}$ & $\mathrm{ND}^{*}$ & 1.4 & 5.0 & 415 & 2.4 & 157 & 0.16 \\
\hline M8-20 & $8-10 \mathrm{~nm}$ & 12 & 13.1 & 20.1 & 6 & 5.2 & 65 & 0.11 \\
\hline M8-25 & $8-10 \mathrm{~nm}$ & 15 & 15.7 & 25.0 & 10 & 5.0 & 73 & 0.17 \\
\hline M8-30 & $8-10 \mathrm{~nm}$ & 18 & 18.6 & 29.7 & 19 & 5.0 & 87 & 0.25 \\
\hline M10-20 & $10-15 \mathrm{~nm}$ & & $+1^{\mathrm{b}}$ & $20^{\mathrm{b}}$ & 9 & 16.7 & 109 & 0.14 \\
\hline
\end{tabular}

bcalculated value (experimental value not determined), ${ }^{\mathrm{c}}$ supporting info, ${ }^{\mathrm{d}}$ from (Eq. $2 \mathrm{~b}$ ).

Measured iron contents in the hybrid particles are in good agreement with the targeted FWR. Moreover, both methods - fit 5 of the whole absorption spectrum of intact HNPs or colorimetric assay of the $\mathrm{FeCl}_{6}{ }^{3-}$ complex content after acidic degradation gave similar results within their respective experimental uncertainties (Table 1 and 2, columns 3 and 4).

Figure 3 shows representative bright field TEM images of 10 PEG- $b$-PBLG (a) and PTMC- $b$-PGA (b) HNPs loaded with $10 \mathrm{wt} \%$ FWR of $10-15 \mathrm{~nm}$ USPIOs, where their respective micellar and vesicular geometry are clearly evidenced. For M1010 hybrid micelles (a), around 1 to 10 USPIOs per particle are fully wrapped by the polymer material and located in the 15 hydrophobic core of the objects. On the contrary, in the case of V10-10 (b), the location of the iron oxide NPs solely at the periphery of the objects confirms their incorporation within the membrane of polymersomes, this behavior being identical for V6-10 vesicles which are loaded at the same FWR but with the 20 smallest USPIOs (Figure 4.a).
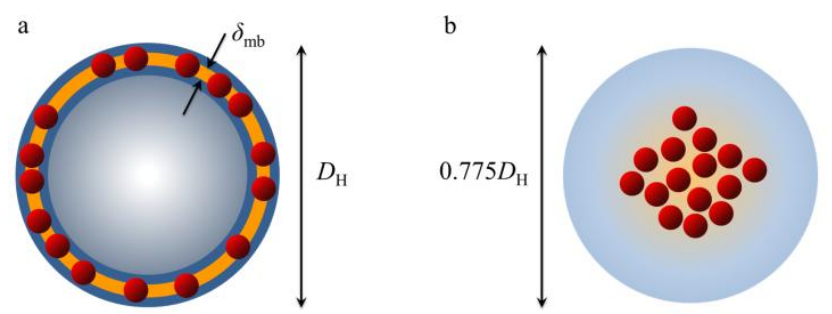

Fig.1 Sketches of the two geometries of hybrid particles, respectively hollow vesicle with USPIOs (red spheres) embedded in the membrane (a) and filled micelle with a hydrophobic (orange) and magnetic core

25 surrounded by a hydrophilic shell (blue) (b). For both geometries, we took into account the theoretical factor between the hydrodynamic and the physical sizes (respectively 1 for a spherical shell and 0.775 for a filled sphere. $^{32,33}$

At low FWRs, USPIOs do not cover the entire surface of the 30 vesicles (Figure 3.b \& 4.a) and form patches, letting some areas uncovered. ${ }^{30}$ Looking at the TEM images of much highly loaded vesicles (Figure 4.b), it appears that the maximum FWR accessible before the destabilization of the system, $70 \mathrm{wt} \%$, also corresponds to the saturation of the membrane where USPIOs are 35 closely packed, leaving very few uncovered areas.

Moreover, as observed by Hickey et al., ${ }^{34}$ the incorporation of USPIOs in the membrane of polymersomes visibly affects their mechanical properties. Indeed, at low FWR, vesicles are spread on the TEM grid and their apparent diameter is higher than the 40 diameter measured by DLS. On the contrary, at high FWR like in the V6-70 sample, nanoparticles rigidify the vesicles that maintain their spherical shape (Figure 4).

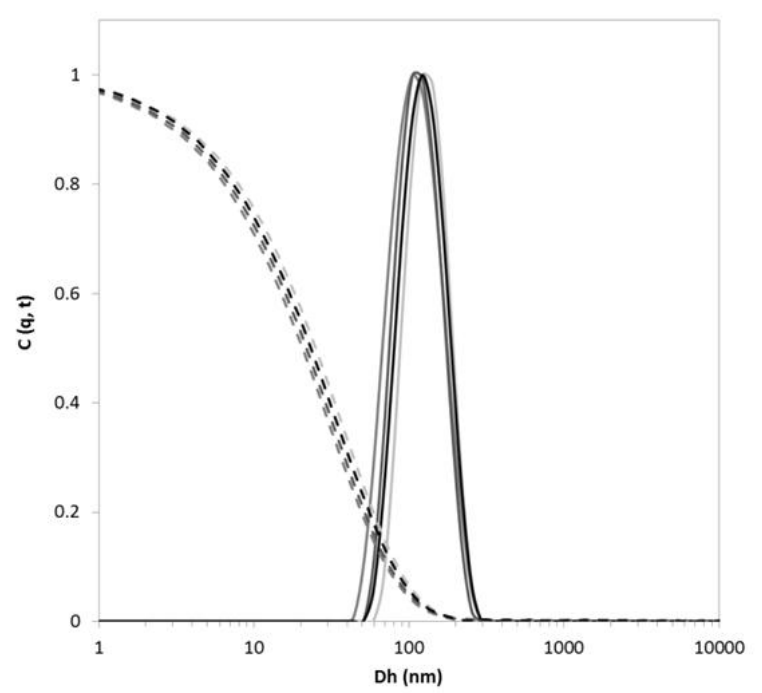

45 Fig.2 Autocorrelation curves and corresponding size distributions obtained by DLS analysis $\left(90^{\circ}\right.$ angle) for hybrid PTMC- $b$-PGA vesicles loaded with 6-7 nm USPIOs at different FWRs. From light grey to black lines: 5, 10, 50 and $70 \%$ FWR. 
The number of USPIOs per hybrid particle can be estimated roughly using the following formulas involving experimental parameters such as the hydrodynamic diameter of the hybrids, $D_{\mathrm{H}}$, membrane thickness, $\delta_{\mathrm{mb}}$ (vesicles only), FWR, and using 5 simple geometric considerations (Figure 1):

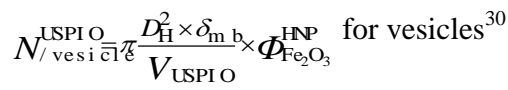

$$
\begin{aligned}
& N_{/ \mathrm{m} \mathrm{i} \mathrm{c} \text { c }}^{\mathrm{USP}} \frac{\pi}{6} \frac{\left(0.775 D_{\mathrm{H}}\right)^{3}}{V_{\text {USPIO }}} \times \Phi_{\mathrm{Fe}_{2} \mathrm{O}_{3}}^{\mathrm{HNP}} \text { for micelles }
\end{aligned}
$$

involving also the volume fraction occupied by iron oxide in the composite and the weight-average volume of the USPIOs: ${ }^{23}$

10

$$
\begin{gathered}
\Phi_{\mathrm{Fe}_{2} \mathrm{O}_{3}}^{\mathrm{HNP}}=\frac{\mathrm{FWR} \times \rho_{\mathrm{copo}}}{\mathrm{FWR} \times \rho_{\mathrm{copo}}+\rho_{\mathrm{Fe}_{2} \mathrm{O}_{3}}} \\
V_{\text {LSPIO }}=\frac{\pi}{6} d_{\mathrm{w}}^{3}
\end{gathered}
$$

where $\rho_{\mathrm{Fe} 2 \mathrm{O} 3}=5 \mathrm{~g} \cdot \mathrm{cm}^{-3}$ and $\rho_{\text {copo }}$ are the mass densities of respectively the inorganic and the organic parts of the HNPs, with $\rho_{\text {copo }}=1 \mathrm{~g} \cdot \mathrm{cm}^{-3}$ for PTMC- $b$-PGA and $\rho_{\text {copo }}=1.28 \mathrm{~g} \cdot \mathrm{cm}^{-3}$ for PEG$15 b$-PBLG (density of PBLG reported in literature, ${ }^{35}$ which appears to be close to that of PEG). Values calculated from Eq. 2a and Eq. $2 b$ are reported in Table 1 and Table 2, respectively. These estimates are pretty well correlated with the approximate numbers of USPIOs per object counted on the TEM pictures.

20
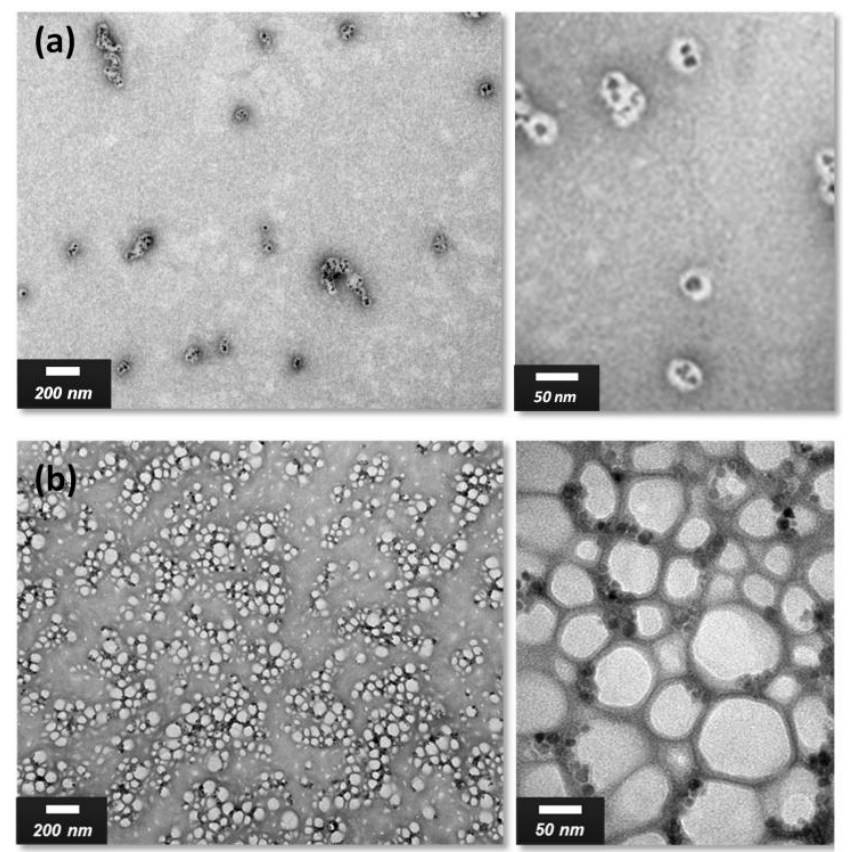

Fig.3 Transmission electron microscopy images of (a) PEG- $b$-PBLG and (b) PTMC- $b$-PGA HNPs loaded with $10 \mathrm{wt} \%$ FWR of $10-15 \mathrm{~nm}$ USPIOs. Negative staining: $1 \%$ uranyl acetate. The approximate numbers of 25 USPIOs per object calculated by (Eq.2a) are respectively 5 per M10-10 micelle (a) from (Eq. 2b) and 2 per V10-10 vesicle (b) from (Eq. 2a), in agreement with the TEM pictures.

\section{${ }_{30} T_{1}$ and $T_{2}$ relaxivities of hybrid copolymer/iron oxide particles}

In the following, we aim at separating the intrinsic relaxometric properties of the USPIOs (due to their weight-average core size $d_{\mathrm{w}}$ and specific magnetization $m_{\mathrm{spe}}$ as individual nanoparticles) and the benefits of their self-assembly into a cluster of given 35 geometry, either as filled micelles or hollow vesicles. The USPIO's size is indeed directly correlated to relaxometric properties for two reasons. At first, the saturation magnetization of USPIOs - hence their $T_{1}$ and $T_{2}$ reducing capability decreases for lower sizes due to the increase of surface-to-volume 40 ratio that reduces the number of atoms that contribute efficiently to the particle's magnetic moment. ${ }^{36}$ In other words, smaller USPIOs are less magnetized (in terms of volume magnetization, that is nothing else than the volume concentration of Bohr magnetons within the magnetic core), a tendency appearing 45 clearly on the data reported in Supporting Information for the three USPIO batches (Table SI-I). The second reason is related to the so-called "outer sphere" mechanism that is invoked generally to explain the higher relaxation rates of protons in the vicinity of superparamagnetic CAs. ${ }^{13,14,}$ 37-39 In this theory also called 50 "Motional Averaging Regime" (MAR), the relaxation rates $R_{\mathrm{i}}=1 / T_{\mathrm{i}}(i=1,2)$ are estimated from the fluctuations of the interaction between the magnetic particle considered as static and the water protons exploring by translational diffusion all the values of dipolar magnetic field lines around the particle. The ${ }_{55}$ parameters are the volume magnetization $M_{\mathrm{v}}$ and the sphere radius $R_{\mathrm{NMR}}$, which is the minimal approach distance between the protons and the centre of the particle, thus $R_{\mathrm{NMR}}$ includes the thickness of any organic coating impermeable to water around the particle. A characteristic time $\tau_{\mathrm{D}}$ of proton diffusion is defined as ${ }_{60} \tau_{\mathrm{D}}=\left(R_{\mathrm{NMR}}\right)^{2} / D$ where $D$ stands for the diffusion constant of the water molecules (that slightly varies with temperature). As long as the particle keeps a high value of $M_{\mathrm{v}}$, increasing the outer shell radius $R_{\mathrm{NMR}}$ leads to a longer time during which the proton interacts with the magnetic field created by the particles. Larger ${ }_{65}$ USPIOs should thus bring higher intrinsic relaxometric properties to the hybrid particle. But from the previous structural study by TEM and DLS, the maximal FWR - hence the number of USPIOs per object that can be accommodated within the selfassembled polymer structures - is much lower for larger 70 magnetic cores: $20 \mathrm{wt} \%$ for $10-15 \mathrm{~nm}$ USPIOs $v s .70 \mathrm{wt} \%$ for $6-$ $7 \mathrm{~nm}$ USPIOs in the case of vesicles. Consequently, it appeared of paramount importance to determine on the one hand which morphology (vesicle or micelle), and on the other hand which parameter (USPIO individual size or USPIO FWR) lead to the 75 assemblies with the best relaxometric properties.

Figure 5 shows typical examples of NMRD longitudinal relaxivity curves $\left(r_{1} v s . v\right)$ obtained for hybrid micelles with the smallest USPIOs (M6-5, Figure 5.a) and the largest ones (M1020, Figure 5.b). Both profiles are flat for low frequencies, then 80 reach a maximum and finally decrease rapidly at higher frequencies. These two examples are typical of all NMRD $T_{1}$ profiles of micelles (Figure SI-6 in Supporting Information), with the peak of $r_{1}(v)$ occurring near $8 \mathrm{MHz}$ for 6-7 nm, $4 \mathrm{MHz}$ for 8$10 \mathrm{~nm}$, and $2 \mathrm{MHz}$ for $10-15 \mathrm{~nm}$ USPIOs, respectively. All 85 samples show a profile qualitatively similar to Endorem ${ }^{\circledR}$, except for a shift of the peak towards lower frequencies and with an increased height when increasing the diameters of the USPIOs. 

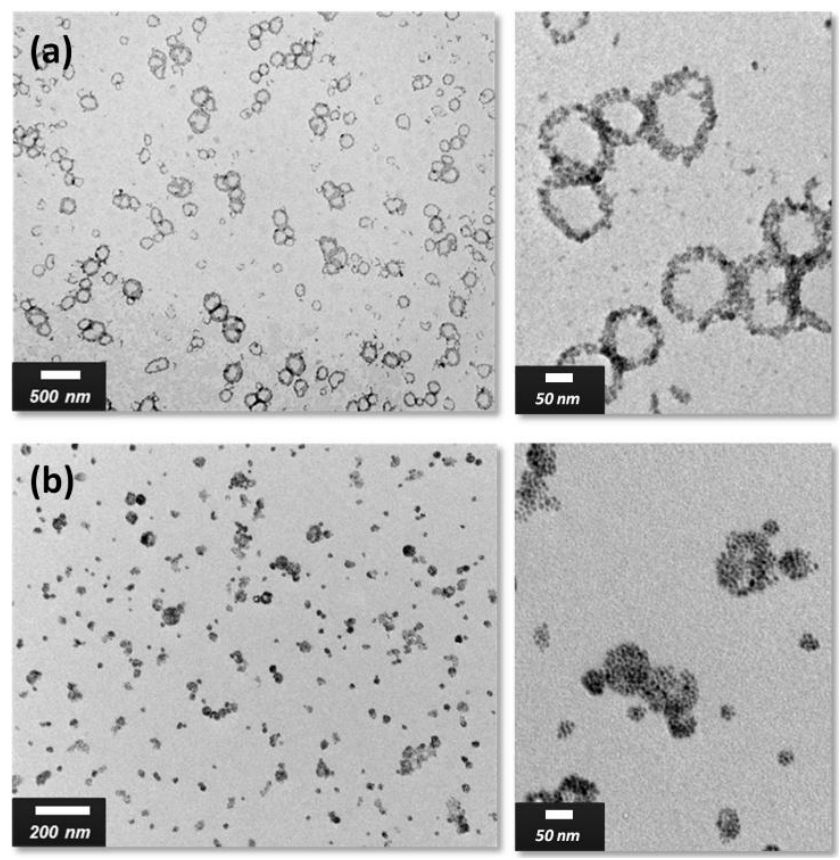

Fig.4 Transmission electron microscopy images of PTMC- $b$-PGA HNPs loaded with (a) $10 \mathrm{wt} \%$ and (b) $70 \mathrm{wt} \%$ FWR of $6-7 \mathrm{~nm}$ USPIOs. The approximate numbers of USPIOs per vesicle calculated by (Eq. 2a) are 5 respectively $\approx 30$ for V6-10 (a) and $\approx 235$ for V6-70 (b), in agreement with the TEM pictures.

The shape of these $r_{l}(v)$ NMRD curves can be explained through the known mechanisms of nuclear longitudinal relaxation in superparamagnetic particles. ${ }^{13,14}$ The first mechanism is the 10 Néel relaxation, dominating at low frequency, induced by the reversal of the magnetic moments through the anisotropy energy barrier. The second mechanism is the Curie relaxation that predominates at higher frequencies and is ascribed to the progressive orientation of the magnetic moments with an 15 increasing field. According to the Langevin's law, this mechanism is a function of the USPIOs' parameters $d_{\mathrm{w}}$ and $m_{\text {spe }}$ and temperature. While Néel's mechanism flattens the curve at low frequencies, Curie's mechanism is responsible for the maximum of $r_{1}(v)$ at higher frequencies. In particular, the 20 inflection point at the right foot of the peak corresponds to $v \tau_{\mathrm{D}} \approx 1$. Consequently, the shift of the peak from $8 \mathrm{MHz}$ for the 6-7 nm USPIOs to $2 \mathrm{MHz}$ for the $10-15 \mathrm{~nm}$ USPIOs is directly explained by the two-fold size increase of the USPIOs' size (thus, respective values of $\tau_{\mathrm{D}}$ varying in a ratio $\sim 4$ ).

25 From a theoretical point of view, the longitudinal relaxivity can be written following a heuristic model first introduced by Roch $e t a l^{37-39}$. The fitting expression of $r_{1}(v)$ reported by Laurent et al. in a popular review paper $^{14}$ (Eq. (27)) has been obtained by linear combination of the cases of null and infinite anisotropy and 30 by approximation of a more complete model, which starts from the spin Hamiltonian, ${ }^{37-39}$ but is not applicable to particles with diameters higher than 5-6 $\mathrm{nm}$ due to un-practicable calculation times.

With the above heuristic model, we fitted only the data of 35 micelle-like samples, as the vesicular samples would require a more refined model due to the particular shell topology of the magnetic part. The simulated curves reported on Figure 5 fit relatively well the experimental NMRD profiles. It should be noted that the values of specific saturation magnetization $m_{\text {spe }}$ 40 resulting from the fits are very close to those measured by magnetometry. From the fitting parameters of Figure 5, we see that the value $\tau_{\text {Néel }}=17 \mathrm{~ns}$ deduced for the $6-7 \mathrm{~nm}$ USPIOs is typical of superparamagnetic iron oxide. The value $\tau_{\text {Néel }}=47 \mathrm{~ns}$ for the 10-15 nm USPIOs is obviously underestimated, since the 45 dispersion of $r_{1}(v)$ at low frequency is too weak to get a reliable estimate of $\tau_{\text {Néel }}$ from the fit. ${ }^{14}$ Another interpretation could be the strong inter-USPIO interactions in these dense micelles, lowering $\tau_{\text {Néel }}$ compared to individual USPIOs. Concerning the distance of minimum approach of the protons, the values of $2 R_{\mathrm{NMR}}$ (11.2 and ${ }_{50} 20.1 \mathrm{~nm}$, respectively) are larger than the weight-average diameters of the magnetic cores $d_{\mathrm{w}}(7.5$ and $14.8 \mathrm{~nm}$, respectively), but remain well below the hydrodynamic diameter $D_{\mathrm{H}}$ values (Table 2). The diffusion constant of water molecules obtained from the fits are twice as lower than the value in the 55 bulk, $D\left(\mathrm{H}_{2} \mathrm{O}\right)=3 \times 10^{-9} \mathrm{~m}^{2} \cdot \mathrm{s}^{-1}$ at $37^{\circ} \mathrm{C}$. The decrease of $D\left(\mathrm{H}_{2} \mathrm{O}\right)$ can be ascribed simply to an excluded-volume effect experienced by water molecules near hydrophobic solutes, ${ }^{40,41}$ which hampers the rotations around hydrogen bonds and divides the water diffusion constant by a geometrical factor around 2 . Therefore we 60 can conclude that the PBLG cores of the micelles are partially penetrated by water molecules.
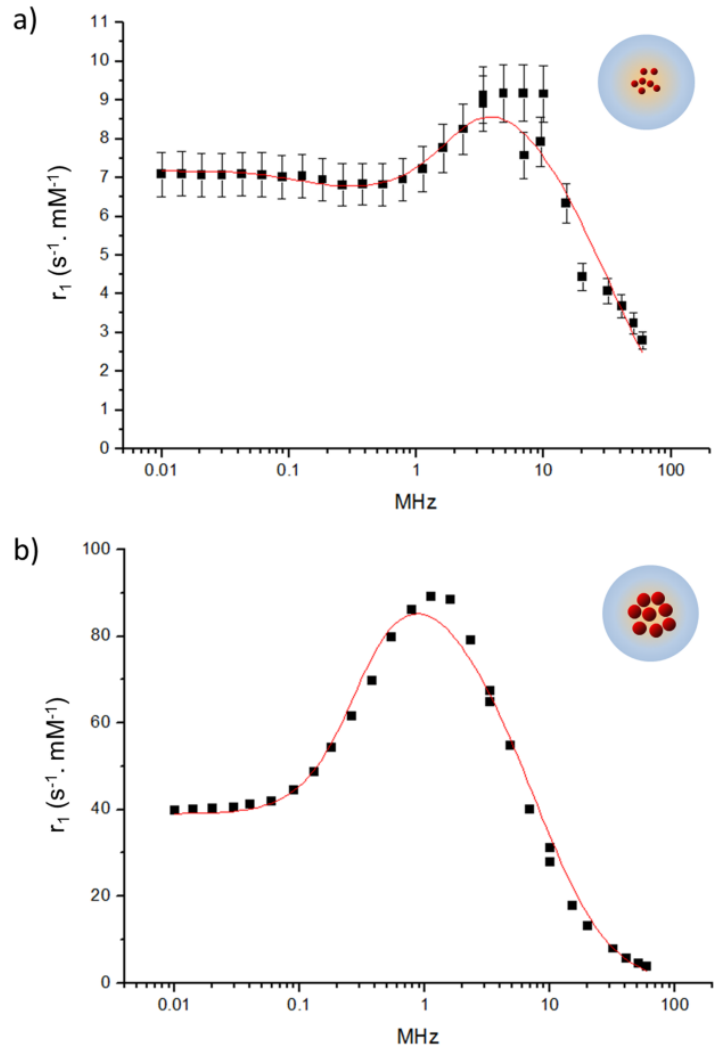

Fig.5 Fitting of the $T_{1}$ NMRD curves with the "outer shell" model for the 65 micelle samples M6-5 (a) and M10-20 (b). The parameters of the fits are: a) $R_{\mathrm{NMR}}=5.6 \mathrm{~nm}, m_{\mathrm{spe}}=51 \mathrm{emu} \cdot \mathrm{g}^{-1}, D\left(\mathrm{H}_{2} \mathrm{O}\right)=2 \times 10^{-9} \mathrm{~m}^{2} \cdot \mathrm{s}^{-1}$, and $\tau_{\text {Néel }}=17 \mathrm{~ns}$; b) $R_{\mathrm{NMR}}=10.1 \mathrm{~nm}, \quad m_{\mathrm{spe}}=67 \mathrm{emu} \cdot \mathrm{g}^{-1}, \quad D\left(\mathrm{H}_{2} \mathrm{O}\right)=1.5 \times 10^{-9} \mathrm{~m}^{2} \cdot \mathrm{s}^{-1}, \quad$ and $\tau_{\text {Néel }}=47 \mathrm{~ns}$.

For transverse relaxivities, the analysis of $1 / T_{2}$ data for the 70 PTMC- $b$-PGA vesicles was complicated by a non monoexponential behavior of the decay of the transverse nuclear magnetization $M_{\mathrm{xy}}$. 


\section{Journal of \\ Materials Chemistry B}

Cite this: DOI: 10.1039/C3TB00429E

a)

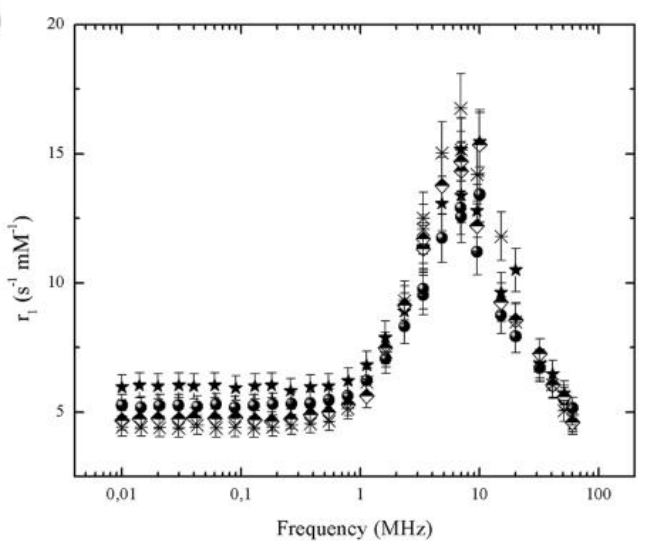

c)

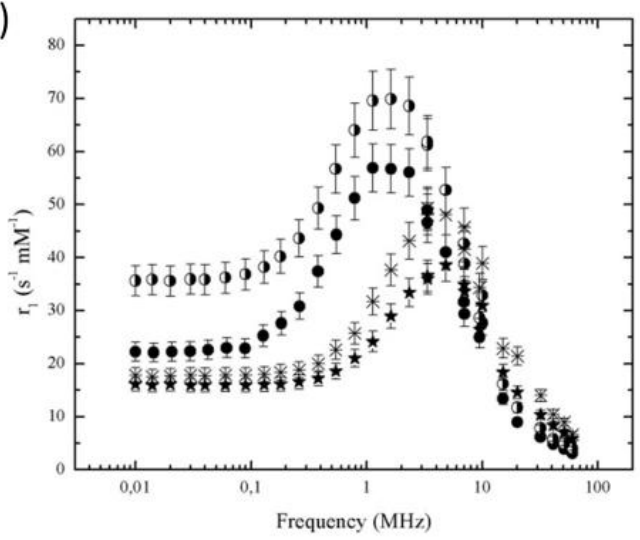

b)
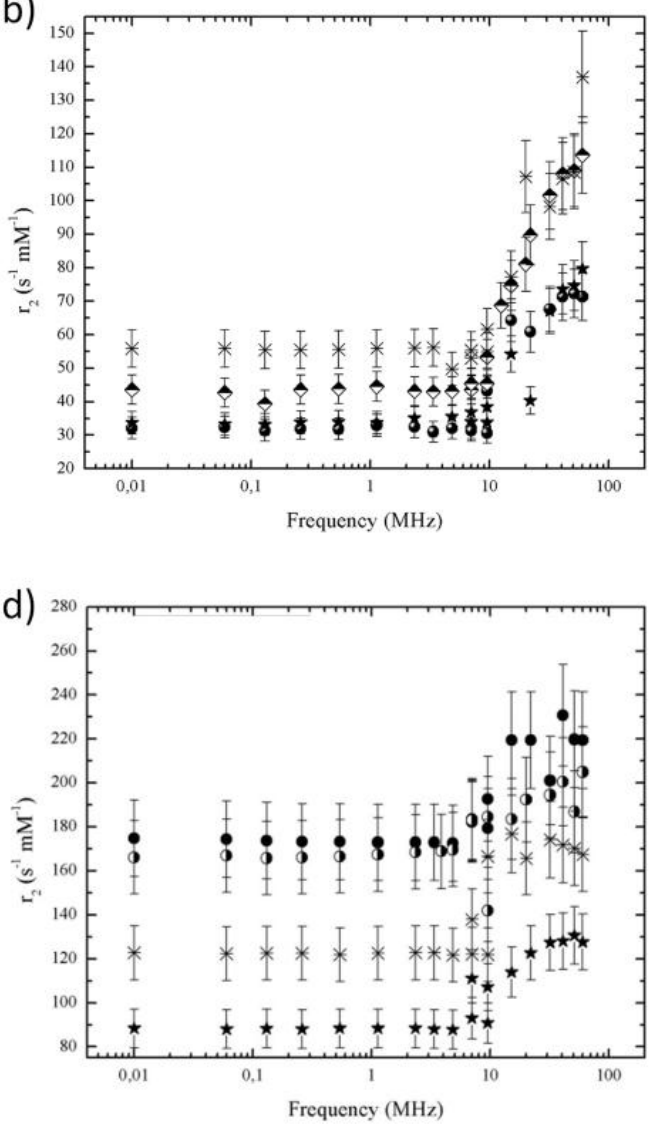
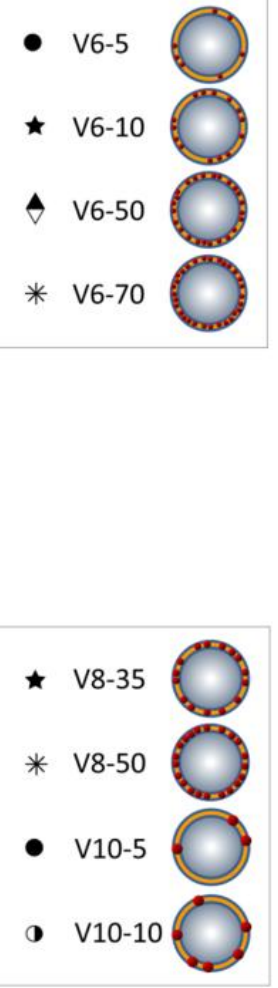

Fig.6 Longitudinal $r_{1}$ (left) and transverse $r_{2}$ (right) relaxivities for PTMC- $b$-PGA HNPs at different USPIOs' diameter and FWRs. For a given USPIOs' size, the $r_{1}(v)$ curves are roughly superimposed, being only slightly translated vertically when increasing the FWR. On the contrary the $r_{2}(v)$ curves vary much more significantly with the FWR. Like with the micelles, at high fields/frequencies $r_{2}$ reaches a plateau for vesicles loaded with 8-10 nm or 10$515 \mathrm{~nm}$ USPIOs, while it continues to increase with the 6-7 nm ones.

In fact, for all these samples, at frequencies higher than $10 \mathrm{MHz}$, two exponentials contribute to the total transverse nuclear magnetization, corresponding to two different nuclear transverse relaxation times $T_{2}$. The fast $T_{2}$ relaxation mode might be 10 ascribed to the contribution of water protons located inside the vesicles, whose diffusion is confined and which are thus maintained at close vicinity of the USPIOs. Since the shortest $T_{2}$ was always lower than 1-3 ms and considering that in standard clinical MRI $T_{2}$-weighted sequences the parameters which affect 15 the signal intensity (repetition time, $T R$, and echo time, $T E$ ) are usually longer than 1-5 ms, we reported on Figure 6 only the relaxivity $r_{2}(v)$ values obtained from the slow $T_{2}$ relaxation time, which ranges from 4 to $60 \mathrm{~ms}$.

Based on the measured $T_{1}$ and the long $T_{2}$ components at the 20 clinical relevant frequency $v=60 \mathrm{MHz}$ (Table 3), micellar and vesicular samples loaded approximately at the same FWR with
USPIOs of the same size such as M8-30 and V8-35 are relatively equivalent in term of $r_{2}$ and $r_{2} / r_{1}$. However when looking at the full curves of transverse relaxivity $r_{2}(v)$, the two geometries 25 respectively PEG- $b$-PBLG micelles (Figure SI-6) and PTMC- $b$ PGA vesicles (Figure 6) display somehow different behaviours. Indeed, while HNPs loaded with the smallest USPIOs' size (M65, V6-5, V6-10, V6-50, V6-70) show a continuous increase of $r_{2}(v)$ at high frequencies ( $v \geq 10 \mathrm{MHz}$, Figure 6.b), all the other 30 samples (M8-20, M8-25, M8-30, M10-20, V8-35, V8-50, V10-5, and $\mathrm{V} 10-10$ ) reach a saturation plateau at frequencies $\mathrm{v} \geq 32 \mathrm{MHz}$, i.e. magnetic field values above $0.75 \mathrm{~T}$ (Figure 6.d). These two behaviours could be ascribed either to individual magnetic properties of the USPIOs or to the global morphology of the 35 clusters: as seen on the magnetization curves (Figure SI-2), at $0.75 \mathrm{~T}$ and above, the magnetization of $8-10 \mathrm{~nm}$ and $10-15 \mathrm{~nm}$ USPIOs are saturated at more than $95 \%$, whereas the 
magnetization of 6-7 nm USPIOs continue to grow, according to Langevin's superparamagnetism law. Since the transverse relaxivity is related to the square of particles' magnetization, ${ }^{13}, 14$ the saturation of $r_{2}$ for M8 and M10 micelles and on the contrary 5 the continuous increase with the M6 ones are totally understood by the differences of USPIOs' size and thus of the saturation degree of the magnetic moments in the corresponding magnetic field values. But in the case of vesicles (Figure 6), the Langevin's function alone cannot explain the $r_{2}(v)$ profiles, since $r_{2}$ continue 10 to grow for $v \geq 10 \mathrm{MHz}(B \geq 0.24 \mathrm{~T})$ not only for $\mathrm{V} 6$ vesicles but also for the V8 and V10 ones, whereas 8-10 nm and 10-15 nm USPIOs are saturated at more than $85 \%$ at these fields. A plausible explanation for this discrepancy between NMRD profiles of micelles and vesicles could be that, unlike micelles,
15 magnetic shells become anisotropic in strong magnetic fields. Neutron scattering experiments reported on similar magnetic polymersomes showed that they deform indeed under magnetic field intensities as low as $B=0.1 \mathrm{~T}(v=4 \mathrm{MHz}) .{ }^{19}, 30$ Thus the continuous increase of the $r_{2}(v)$ profiles could be due to the 20 combined effects of the magnetization curve of the USPIOs (until the total moment saturates) and the deformation of the vesicles that continue to deform further under increasing fields. The non spherical symmetry of such soft magnetic shells under a strong applied magnetic field should be deeper investigated in order to ${ }_{25}$ try fitting the full experimental $T_{2}$ NMRD profiles, but this is beyond the scope of the present article.

Table 3 Intra-aggregate volume fractions and magnetizations, estimates and measured values of $r_{2}$ and $r_{2} / r_{1}$ at $60 \mathrm{MHz}$.

\begin{tabular}{|c|c|c|c|c|c|}
\hline Sample & $\Phi_{\text {intra }}$ & $\begin{array}{c}M_{\mathrm{v}} \\
\left(\mathbf{A} \cdot \mathbf{m}^{-1}\right)\end{array}$ & $\begin{array}{c}r_{2} \text { predicted value } \\
\text { from MAR or SDR } \text { model }^{\mathrm{a}} \\
\left(\mathrm{s}^{-1} \cdot \mathrm{mM}_{\mathrm{Fe}}^{-1}\right) \\
\end{array}$ & $\begin{array}{c}r_{2} \text { measured at } \\
60 \mathrm{MHz}(1.41 \mathrm{~T}) \\
\left(\mathrm{s}^{-1} \cdot \mathrm{mM}_{\mathrm{Fe}}^{-1}\right) \\
\end{array}$ & $\begin{array}{c}r_{2} / r_{1} \\
\text { at } 60 \mathrm{MHz}\end{array}$ \\
\hline V6-5 & $0.5 \%$ & $1.4 \times 10^{3}$ & 67 (MAR) & 71 & 14 \\
\hline V6-10 & $1.1 \%$ & $3.3 \times 10^{3}$ & 108 (MAR) & 80 & 17 \\
\hline V6-50 & $4.9 \%$ & $1.4 \times 10^{4}$ & $\sim 120$ (SDR) & 114 & 25 \\
\hline V8-35 & $5.8 \%$ & $1.8 \times 10^{4}$ & $\sim 140$ (SDR) & 128 & 22 \\
\hline V8-50 & $6.8 \%$ & $2.1 \times 10^{4}$ & $\sim 180$ (SDR) & 167 & 25 \\
\hline V10-5 & $7 \%$ & $2.2 \times 10^{3}$ & 75 (MAR) & 219 & 71 \\
\hline V10-10 & $1.6 \%$ & $5.3 \times 10^{3}$ & 125 (MAR) & 205 & 51 \\
\hline V10-20 & $1.5 \%$ & $5.5 \times 10^{3}$ & $\sim 270($ SDR $)$ & 280 & 103 \\
\hline M6-5 & $1.2 \%$ & $3.5 \times 10^{3}$ & $\sim 170$ (SDR) & 180 & 90 \\
\hline M8-20 & $4.8 \%$ & $1.5 \times 10^{4}$ & $\sim 125$ (SDR) & 95 & 20 \\
\hline M8-25 & $5.9 \%$ & $1.9 \times 10^{4}$ & $\sim 135$ (SDR) & 90 & 19 \\
\hline M8-30 & $7 \%$ & $2.2 \times 10^{4}$ & $\sim 140$ (SDR) & 105 & 30 \\
\hline M10-20 & $4.8 \%$ & $1.7 \times 10^{4}$ & $\sim 400$ (SDR) & 500 & 126 \\
\hline Endorem $^{\circledR}$ & $26 \%$ & $7.3 \times 10^{4}$ & $\sim 96$ (SDR) & 98 & 10 \\
\hline
\end{tabular}

${ }^{a}$ The intra-aggregate volume fraction occupied by $\gamma-\mathrm{Fe}_{2} \mathrm{O}_{3}$ was used to calculate the magnetization over the whole object (micelle or vesicle) and to 30 estimate the transverse relaxivity limit at high frequency within the frame of the MAR model of protons spins relaxation. Other values come from MonteCarlo simulations of the static dephasing regime (SDR) for peculiar values of $M_{\mathrm{v}}{ }^{23,42}$

On the structural point of view, there is a discrepancy between micelles and vesicles in term of the "intra-aggregate" volume fraction occupied by iron oxide inside the HNP. ${ }^{23}$ For the 35 micelles which internal core is filled with USPIOs and only marginally permeable to water, the volume fraction is identical to the dry volume fraction given by Eq. (3) and measurable by TGA. For vesicles, the internal aqueous core needs to be taken into account to estimate the effective intra-aggregate volume 40 fraction through $\Phi_{\text {intra }}=\Phi^{\mathrm{HNP}} \times 6 \delta_{\mathrm{mb}} / D_{\mathrm{H}}$, as reported on column two of Table 3. Effective intra-aggregate volume fractions $\Phi_{\text {intra }}$ are needed in order to calculate the volume magnetizations at saturation $M_{\mathrm{v}}=\Phi_{\text {intra }} \times m_{\text {spe }}$ of the entire HNPs. From these values, it is possible to compute estimated $r_{2}$ of the HNPs using either the 45 MAR theory (for $D_{\mathrm{H}}$ values sufficiently small) or Monte-Carlo simulations when the size of the clusters falls in the validity range of another model called "static dephasing regime" (SDR), as shown in recent studies intended to rationalize the comparison between experimental $r_{2}$ values and the available models. ${ }^{23,42}$
50 In practical, for the smaller HNPs we used the scaling law $r_{2} \cdot \Phi_{\text {intra }} \sim 11.6 \times 10^{-12} \cdot\left(M_{\mathrm{v}} \cdot D_{\mathrm{H}}\right)^{2}$, characteristic of the MAR model. ${ }^{23}$ For micelles or vesicles that are either larger or more magnetic, we used the results of Monte Carlo simulations made in the closest range of magnetization $M_{\mathrm{v}}$ (see Figure 2 in Vuong et ${ }_{55} \mathrm{al}^{23}$ ); this method yields less precise results since simulations of the curve $r_{2} \cdot \Phi_{\text {intra }} v s . D_{\mathrm{H}}$ are needed for each value of $M_{\mathrm{v}}$ Nevertheless, the models (either MAR or SDR) lead to estimates of the high field transverse relaxivity that are reasonably close to the experimental data (Table 3), a plausible source of discrepancy 60 being the size-dispersity, suggesting the possibility in future of improved theory-experiment matching by size grading. Another indicator of the relaxometric properties of a given MRI CA is the ratio between the transverse and longitudinal relaxation rates $\left(r_{2} / r_{1}\right)$, being $\sim 1-2$ in the case of traditional paramagnetic ${ }_{65}$ (positive) CAs and above 10 and up to 50 or more in the case of superparamagnetic (negative) CAs. ${ }^{13}$ Negative CAs with a high $r_{2} / r_{1}$ ratio enable obtaining contrasted images of tissues with $T_{2^{-}}$ 
weighted or $T_{2}{ }^{*}$-weighted MRI sequences. For both particle types, the measured $r_{2} / r_{1}$ ratios (Table 3 , column 6) are always greater than 10 , which is the value obtained for the negative contrast agent Endorem ${ }^{\circledR}$. Two samples (V10-20, M10-20) elicit 5 remarkably higher values, one order of magnitude above the Endorem $^{\circledR}$, suggesting an optimal configuration with the most strongly magnetized $10-15 \mathrm{~nm}$ USPIOs confined inside a hydrophobic environment that is either a spherical core for PEG$b$-PBLG micelles or between the leaflets of a bilayer membrane 10 for PTMC- $b$-PGA vesicles.

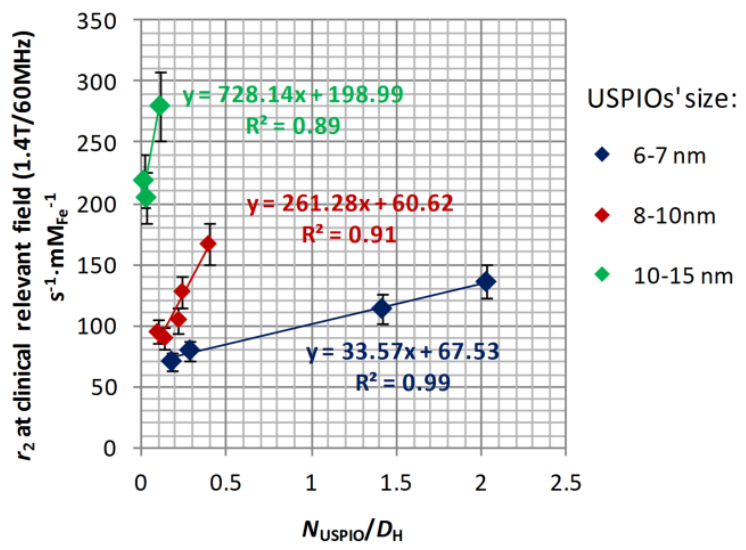

Fig. 7 Master curve representing the relavixity $r_{2}$ at high field $(60 \mathrm{MHz}$ for the proton resonance) $v s$. the ratio of the number of USPIOs divided by the hydrodynamic diameter for the majority of samples reported in this 15 study, both vesicles (V6-5, V6-10, V6-50, V6-70, V8-35, V8-50, V10-5, V10-10, V10-20) and micelles (M8-20, M8-25, M8-30).

Then, we tentatively aimed at deciphering which one from USPIOs' size, FWR, and micelle vs. vesicle geometry is the most influent parameter to increase the transverse relaxivity. We show 20 here that for a given size of iron oxide core, the value of $r_{2}$ at $60 \mathrm{MHz}$ depends mainly on the number of encapsulated USPIOs $\left(N_{\text {USPIO }}\right)$ divided by the diameter of the cluster $\left(D_{\mathrm{H}}\right)$. This parameter is relevant not only for relaxometry, but also to estimate the magnetophoretic velocity of magnetic objects in a ${ }_{25}$ field gradient. ${ }^{30}$ In this case, the morphology (vesicle or micelle) has only a minor influence, as shown on Figure 7 where $r_{2}$ is plotted $v s$. $N_{\mathrm{USPIO}} / D_{\mathrm{H}}$, for both types of samples. A linear relation is observed for each size of USPIOs, independently of the HNP morphology (all the data overlap in case of the 8-10 nm USPIOs). 30 Indeed, the parameter that has the greatest influence on the final relaxivity of the assemblies is the individual size of the USPIOs. For a given USPIOs' size range, $r_{2}$ is related to both the external diameter $D_{\mathrm{H}}$ and the intra-aggregate volume fraction of magnetic material $\Phi_{\text {intra }}$, which variation law with the FWR used during the 35 synthesis is dependent upon the geometry. But when designing magnetic clusters, an easy thumb-rule for the chemists consists in increasing the number of USPIO per cluster $\left(N_{\text {USPIO }}\right)$ while keeping the size of the assemblies $\left(D_{\mathrm{H}}\right)$ at a moderate value.

From these results, one can conclude that the structure of the 40 cluster (micelle or vesicle) has a weak influence on transverse relaxivity, mostly by the shape of the profile of $r_{2} v s$. field or frequency as measured by fast-field cycling. Nevertheless, it was observed in a recent study that the morphology can impact the relaxivity of magnetic assemblies, a particular magneto-core/shell
45 structure exhibiting a higher relaxivity than usual dense magnetomicelles and magneto-polymersomes. ${ }^{34}$ The authors ascribed this phenomenon to a better water accessibility when USPIOs are located at intermediate distance in the corona of the micelles rather than distributed homogeneously or buried deeply in the 50 hydrophobic cores, where they are separated from water protons by a thick shell of hydrophobic polymer, in that case polystyrene.

This particular magneto-core/shell morphology was obtained neither with PTMC- $b$-PGA nor with PEG- $b$-PBLG copolymers, presumably due to a better compatibility between the 55 hydrophobic blocks and the surfactant-coated USPIOs (thus no micro-phase separation in the assemblies). For both morphologies tested in this work (magnetic micelles or magnetic vesicles), the thickness of the non-magnetic layer covering the USPIOs was small enough or sufficiently permeable to water molecules. Thus 60 all magnetic material introduced in the particles efficiently contributes to the measured $r_{2}$. Our results are also in accordance with recent Monte-Carlo simulations of dense spherical micelles and hollow shells of USPIOs' clusters, leading to comparable $r_{2}$ values for both geometries. ${ }^{42}$

65 In this study, the sample maximizing the transverse relaxivity was the batch of M10-20 micelles prepared at high feed weight ratio $(20 \mathrm{wt} \%)$ from the biggest USPIOs $(10-15 \mathrm{~nm})$ clustered in a compact fashion (meaning maximizing the ratio $N_{\mathrm{USPIO}} / D_{\mathrm{H}}$ ). With $r_{2}=500 \mathrm{~s}^{-1} \cdot \mathrm{mM}_{\mathrm{Fe}}^{-1}$, they compete with the samples from the 70 class of magnetic hydrogels, the best $T_{2}$ contrast agents reported so far, whose superior transverse relaxivity was ascribed to their hydrophilic nature. ${ }^{43,44}$ The world record of transverse relaxivity for iron oxide HNPs was recently pushed higher, slightly above $600 \mathrm{~s}^{-1} \cdot \mathrm{mM}_{\mathrm{Fe}}{ }^{-1}$ at $1.4 \mathrm{~T}$ for a series of USPIOs clusters chemically 75 cross-linked by hydrophilic polymer chains. ${ }^{45}$ Nevertheless, this type of high $r_{2}$ magnetic hydrogels follows the MAR model: the universal scaling law ${ }^{23} r_{2} \sim\left(m_{\text {spe }} \cdot D_{\mathrm{H}}\right)^{2} \cdot \Phi_{\text {intra }}$ is still valid, but the volume fraction of iron oxide in the composite measured by TGA (thus in dry state) must be normalized by the swelling ratio of the 80 hydrogel (volume of water divided by volume of solid matter), which is totally equivalent to the interpretation by the authors that the surface-to-surface separation between the USPIOs within the clusters is enlarged, giving space for the entry of water. ${ }^{45}$

\section{Conclusions}

${ }_{85}$ A series of hybrid particles was prepared by self-assembly of amphiphilic copolymers and various amounts of hydrophobically coated USPIOs of three different sizes. Depending on the copolymer used, these HNPs exhibited either a micellar or vesicular morphology. Their relaxometric properties were studied 90 on a large frequency range and results compared to theoretical models. Focusing on geometrical and composition parameters, we found that, for a given USPIO's size, for a fixed frequency $(60 \mathrm{MHz})$ and for the spherical geometry, $r_{2}$ varies linearly with the ratio of the number of embedded USPIOs over the 95 hydrodynamic diameter of the cluster $\left(r_{2} \sim N_{\mathrm{USPIO}} / D_{\mathrm{H}}\right)$, whatever the HNP morphology. This plot also clearly shows that the individual USPIOs' size has the uppermost effect onto the final efficiency of these hybrid NPs as contrast agents. The cluster geometry (micelle or vesicle) has a mild influence on the final 100 properties as contrast agent, but a strong influence on the physical properties, as seen by comparing the transverse relaxivity curves 
$r_{2}(v)$; further efforts to interpret these data quantitatively using more complex models should be done. Thanks to their relatively small size and the nature of the polymer hydrophobic blocks (poly(ester) and poly(amino acid)) used indeed, the particles 5 studied in this work have partially hydrated cores. Therefore, all the magnetic material embedded in the HNPs - be it micelle or vesicle - contributes efficiently to the final contrast agent properties. According to our results, it is likely that the structure of polymer/iron oxide assemblies will have an impact on their 10 efficiency as MRI contrast agents in the case of larger objects prepared with highly hydrophobic material where USPIOs buried in the dehydrated core will not have any impact on water protons. Consequently, for small particles prepared from biodegradable polymers, the choice of geometry can be determined by other 15 requirements, for instance the type of bio-active compounds that needs to be loaded: for a hydrophobic drug (e.g. Paclitaxel), a micelle geometry will be preferred, whereas for a hydrophilic one (e.g. nucleic acids) the encapsulation within the aqueous core of a vesicle would be best suited. When designing nanocarriers for 20 theranostic applications from magnetic polymer HNPs, the choice of nanostructure (spherical micelles, polymersomes, core-shells, hydrogels...) can be dictated by other requirements (drug loading efficiency, grafting of biological ligands for tumor targeting...) since MRI contrast efficiency can always be optimized, whatever 25 the particulate structure, by choosing appropriate magnetic core size, localization and feed weight ratio of the USPIOs, a good hint being to maximize the $N_{\mathrm{USPIO}} / D_{\mathrm{H}}$ ratio.

\section{Acknowledgements}

This work was supported by NANOTHER project (Seventh 30 Framework Programme: Theme NMP-2007, Large Scale Integrating Collaborative Project $\mathrm{n}^{\circ}$ CP-IP 213631-2) and by Italian MIUR project FIRB "RINAME" (RBAP114AMK). Authors also thank RNP-ESF P2M project for support.

\section{Abbreviations}

${ }_{35}$ CA, contrast agent; CPMG, Carr Purcell Meiboom Gill; CT, com-puted tomography; DDS, drug delivery systems; DLS, dynamic light scattering; FFC, fast-field-cycling; FWR, feed weight ratio; HNP, hybrid nanoparticle; MAR, motional averaging regime; MPS, mononuclear phagocyte system; MRI, 40 magnetic resonance imaging; NANOTHER, integration of novel NANOparticle based technol-ogy for THERapeutics and diagnosis of different types of cancer; NMRD, nuclear resonance dispersion; PBLG, poly( $\gamma$-benzyl-L-glutamate); PDI, polydispersity index; PEG, poly(ethylene glycol); PET, positron 45 emission tomography; PGA, poly(L-glutamic acid); PTMC-, poly(trimethylene carbonate); SDR, static dephasing re-gime; SPECT, single-photon emission computed tomography; TEM, transmission electron microscopy; TGA, thermogravimetric analysis; USPIO, ultrasmall superparamagnetic iron oxide; ${ }_{50}$ UUSPIO, ultra-ultrasmall super-paramagnetic iron oxide; VSM, vibrating sample magnetometry; SAED, selected area electron diffraction; Research Network Programme, RNP; European Science Foundation, ESF; P2M, precision polymer materials.

\section{${ }_{55}$ Notes and references}

${ }^{a}$ Dipartimento di Fisica, Università degli Studi di Milano, I-20133 Milano, and Consorzio INSTM, ITALY

${ }^{b}$ Université de Bordeaux/IPB, ENSCBP, 16 avenue Pey Berland, 33607

Pessac Cedex, France

${ }_{60}{ }^{c}$ CNRS, Laboratoire de Chimie des Polymères Organiques (UMR5629),

Pessac, France. Fax: +33 5-4000-8487; Tel: +33 5-4000-3695; E-mail: lecommandoux@enscbp.fr and olivier.sandre@ipb.fr

${ }^{d}$ CNISM, Consorzio INSTM and Università degli Studi di Pavia,

Dipartimento di Fisica, I-27100, Pavia, ITALY. Tel: +39 03-8298-7499; 65 E-mail: alessandro.lascialfari@unipv.it

${ }^{e}$ Dipartimento di Fisica e Astronomia, Università di Bologna, I-40127, Bologna, ITALY

${ }^{f}$ Dipartimento di Chimica, Università di Firenze and Consorzio INSTM, I-50019 Sesto Fiorentino, ITALY

$70{ }^{g}$ ISTM - CNR, I-20133 Milano, ITALY

${ }^{h}$ Istituto nanoscienze - CNR, Modena, ITALY

$\dagger$ Electronic Supplementary Information (ESI) available: SI-1. Synthesis of iron oxide superparamagnetic nanoparticles, SI-2 Size sorting process 75 of the iron oxide superparamagnetic nanoparticles, SI-3. Characterization of the iron oxide nanoparticles dispersions, Magnetization curves, Selected area electron diffraction. Iron oxide concentration determination. Longitudinal and transverse NMRD profiles for magnetic micelles. Field Cooled (FC) and Zero Field Cooled (ZFC) magnetization curves. See 80 DOI: $10.1039 / \mathrm{b} 000000 \mathrm{x} /$

\# These authors contributed equally to this work.

* Corresponding authors.

\section{References}

1. D.-E. Lee, H. Koo, I.-C. Sun, J. H. Ryu, K. Kim and I. C. Kwon, 85 Chemical Society Reviews, 2012, 41, 2656-2672.

2. P. A. Rinck, Blackwell, Oxford, 1993.

3. H. Yim, S. Seo and K. Na, Journal of Nanomaterials, 2011, 2011.

4. W.-Y. Huang and J. J. Davis, Dalton Transactions, 2011, 40, 60876103.

905. Q. A. Pankhurst, N. T. K. Thanh, S. K. Jones and J. Dobson, Journal of Physics D: Applied Physics, 2009, 42, 224001.

6. C. C. Berry, Journal of Physics D: Applied Physics, 2009, 42, 224003.

7. T. Ninjbadgar and D. F. Brougham, Advanced Functional Materials, 2011, 21, 4769-4775.

8. A. J. Cole, V. C. Yang and A. E. David, Trends in biotechnology, 2011, 29, 323-332.

9. S. Laurent, L. Vander Elst, A. Roch and R. N. Muller, in NMR-MRI, $\mathrm{mSR}$ and Mossbauer Spectroscopies in Molecular Magnets, eds. P.

100 Carretta and A. Lascialfari, Springer-Verlag, Italia, 2007, p. 71.

10. A. Lascialfari and M. Corti, in NMR-MRI,mSR and Mossbauer Spectroscopies in Molecular Magnets, eds. P. Carretta and A. Lascialfari, Springer-Verlag, Italia, 2007.

11. U. I. Tromsdorf, O. T. Bruns, S. C. Salmen, U. Beisiegel and H. 105 Weller, Nano Letters, 2009, 9, 4434-4440.

12. B. H. Kim, N. Lee, H. Kim, K. An, Y. I. Park, Y. Choi, K. Shin, Y. Lee, S. G. Kwon, H. B. Na, J.-G. Park, T.-Y. Ahn, Y.-W. Kim, W. K. Moon, S. H. Choi and T. Hyeon, Journal of the American Chemical Society, 2011, 133, 12624-12631.

110 13. Y. Gossuin, P. Gillis, A. Hocq, Q. L. Vuong and A. Roch, Wiley Interdisciplinary Reviews: Nanomedicine and Nanobiotechnology, 2009, 1, 299-310.

14. S. Laurent, D. Forge, M. Port, A. Roch, C. Robic, L. Vander Elst and R. N. Muller, Chemical Reviews, 2008, 108, 2064-2110.

115 15. H. Ai, C. Flask, B. Weinberg, X. T. Shuai, M. D. Pagel, D. Farrell, J. Duerk and J. Gao, Advance Materials, 2005, 17, 1949-1952.

16. J.-F. Berret, N. Schonbeck, F. Gazeau, D. El Kharrat, O. Sandre, A. Vacher and M. Airiau, Journal of the American Chemical Society, 2006, 128, 1755-1761.

120 17. J. R. McCarthy and R. Weissleder, Advanced Drug Delivery Reviews, 2008, 60, 1241-1251. 
18. C. Corot, P. Robert, J.-M. Idée and M. Port, Advanced Drug Delivery Reviews, 2006, 58, 1471-1504.

19. S. Lecommandoux, O. Sandre, F. Chécot, J. Rodriguez-Hernandez and R. Perzynski, Advanced Materials, 2005, 17, 712-718.

5 20. W. Agut, D. Taton, A. Brulet, O. Sandre and S. Lecommandoux, Soft Matter, 2011, 7, 9744-9750.

21. C. W. Jung and P. Jacobs, Magnetic Resonance Imaging, 1995, 13, 661-674.

22. C. W. Jung, Magnetic Resonance Imaging, 1995, 13, 675-691.

10 23. Q. L. Vuong, J.-F. Berret, J. Fresnais, Y. Gossuin and O. Sandre, Advanced Healthcare Materials, 2012, 1, 502-512.

24. R. Massart, Magnetics, IEEE Transactions on, 1981, 17, 1247-1248.

25. R. Massart, E. Dubois, V. Cabuil and E. Hasmonay, Journal of Magnetism and Magnetic Materials, 1995, 149, 1-5.

15 26. C. Da Cruz, O. Sandre and V. Cabuil, The Journal of Physical Chemistry B, 2005, 109, 14292-14299.

27. C. Bonini, L. Heux, J.-Y. Cavaillé, P. Lindner, C. Dewhurst and P. Terech, Langmuir, 2002, 18, 3311-3314.

28. M. E. M. Barbosa, V. Montembault, S. Cammas-Marion, G. Ponchel

20 and L. Fontaine, Polymer International, 2007, 56, 317-324.

29. C. Sanson, C. Schatz, J.-F. Le Meins, A. Brûlet, A. Soum and S. Lecommandoux, Langmuir, 2009, 26, 2751-2760.

30. C. Sanson, O. Diou, J. Thévenot, E. Ibarboure, A. Soum, A. Brûlet, S. Miraux, E. Thiaudière, S. Tan, A. Brisson, V. Dupuis, O. Sandre and S. Lecommandoux, ACS Nano, 2011, 5, 1122-1140.

31. R. Kimmich and E. Anoardo, Progress in Nuclear Magnetic Resonance Spectroscopy, 2004, 44, 257-320.

32. D. Kunz, A. Thurn and W. Burchard, Colloid \& Polymer Science, 1983, 261, 635-644.

30 33. W. Burchard, in Light Scattering from Polymers, Springer Berlin, Heidelberg, 1983, pp. 1-124.

34. R. J. Hickey, A. S. Haynes, J. M. Kikkawa and S.-J. Park, Journal of the American Chemical Society, 2011, 133, 1517-1525.

35. L. Rubatat, X. Kong, S. A. Jenekhe, J. Ruokolainen, M. Hojeij and R. Mezzenga, Macromolecules, 2008, 41, 1846-1852.

36. Y.-w. Jun, Y.-M. Huh, J.-s. Choi, J.-H. Lee, H.-T. Song, S. Kim, S. Yoon, K.-S. Kim, J.-S. Shin, J.-S. Suh and J. Cheon, Journal of the American Chemical Society, 2005, 127, 5732-5733.

37. A. Roch, R. N. Muller and P. Gillis, The Journal of Chemical Physics, 1999, 110, 5403-5411.

38. P. Gillis, A. Roch and R. A. Brooks, Journal of Magnetic Resonance, 1999, 137, 402-407.

39. J. W. M. Bulte, R. A. Brooks, B. M. Moskowitz, L. H. B. Jr and J. A. Frank, Journal of Magnetism and Magnetic Materials, 1999, 194, 217-223.

40. D. Laage, G. Stirnemann and J. T. Hynes, The Journal of Physical Chemistry B, 2009, 113, 2428-2435.

41. D. Laage, G. Stirnemann, F. Sterpone, R. Rey and J. T. Hynes, Annual Review of Physical Chemistry, 2011, 62, 395-416.

50 42. Q. L. Vuong, P. Gillis and Y. Gossuin, Journal of Magnetic Resonance, 2011, 212, 139-148.

43. C. Paquet, H. W. de Haan, D. M. Leek, H.-Y. Lin, B. Xiang, G. Tian, A. Kell and B. Simard, ACS Nano, 2011, 5, 3104-3112.

44. H. W. de Haan and C. Paquet, Magnetic Resonance in Medicine, 2011, 66, 1759-1766.

45. J. S. Riffle, N. Pothayee, S. Balasubramanian, N. Pothayee, N. Jain, N. Hu, Y. Lin, R. M. Davis, N. Sriranganathan and A. P. Koretsky, Journal of Materials Chemistry B, 2013, DOI: 10.1039/C1032TB00275B

60 